\title{
Anesthesia of a dental patient with Angelman syndrome -A case report-
}

\author{
Bo Sung Kim, Jin Seok Yeo, and Si Oh Kim \\ Department of Anesthesiology and Pain Medicine, Kyungpook National University School of Medicine, Daegu, Korea
}

Angelman syndrome is characterized by a partial deficit of paired autosomal chromosome 15, which contains a subunit of the GABA (Gamma-Amino Butyric Acid) receptor. Many drugs that act on the CNS (Central Nerve System) during anesthesia are believed to exert their effects via the GABA receptors. We describe the anesthesia of a 7 year-old female patient with Angelman syndrome who underwent surgery for dental caries. The basic factors that needed to be considered when administering anesthesia to this patient were epilepsy, significant dominance of the vagal tone, craniofacial abnormalities and peripheral muscular atrophy. Inhalational anesthetics (sevoflurane) were employed for this patient. The patient had an uneventful peri-operative period and was discharged home on the same day of the operation. (Korean J Anesthesiol 2010; 58: 207-210)

Key Words: Anesthesia, Angelman syndrome, Chromosome 15, GABA receptor, Outpatient surgery.

Angelman syndrome was first reported by Harry Angelman in 1965 [1]. This syndrome is characterized by bouts of laughter, intellectual retardation, cerebella ataxia, severe developmental delay, malformations of the skull and facial bones, seizures, etc. Such characteristics are due to a partial deficit on a region of paired chromosome 15. The chromosome defect is responsible for the dysfunction of the GABA (Gamma-Amino Butyric Acid) receptor, as well as the damage to the synthesis and secretion of GABA [2-4]. The GABA receptor is a common channel for the action of many drugs used for general anesthesia. Patients with Angelman syndrome have an impairment of this receptor, and need to be placed under general anesthesia even for a simple procedure. The anesthetic problems related to the syndrome involve seizures, hyperfunction of vagus nerves, malformations of the skull and facial bones, peripheral muscular atrophy, etc. We report a case of general anesthesia of a 7 year-old female outpatient with Angelman syndrome for the treatment of dental caries with a review of the relevant literature. This case shows that peripheral muscular atrophy in general anesthesia for outpatients requires more careful attention.

\section{Case Report}

A 7 year-old, $113 \mathrm{~cm}, 17 \mathrm{~kg}$, female patient with tooth decay presented for general anesthesia for tooth conservation day surgery because outpatient treatment was not possible due

Received: July 7, 2009. Revised: 1st, July 16, 2009; 2nd, July 29, 2009. Accepted: August 13, 2009.

Corresponding author: Jin Seok Yeo, M.D., Department of Anesthesiology and Pain Medicine, Kyungpook National University School of Medicine, 200, Dongduk-ro, Jung-gu, Daegu 700-721, Korea. Tel: 82-53-420-5872, Fax: 82-53-426-2760, E-mail: jsyeo@hmail.knu.ac.kr CCThis is an open-access article distributed under the terms of the Creative Commons Attribution Non-Commercial License (http:// creativecommons.org/licenses/by-nc/3.0/), which permits unrestricted non-commercial use, distribution, and reproduction in any medium, provided the original work is properly cited. 
to her lack of cooperation. Her medical history showed that she had been delivered normally after 39 weeks of gestation, weighing $2.8 \mathrm{~kg}$. At birth, the child had a weak suck. Seven months after birth, pediatric parent visits were made due to a general developmental delay, microcephaly, and sleep disorder. Fourteen months after birth, brain MRI (Magnetic Resonance Imaging) was performed, which revealed no abnormalities. However, a chromosomal study showed a deficiency of Chromosome 15 at q11.2-q13, and a FISH (Fluorescence in situ hybridization) test confirmed Angelman syndrome (Fig. 1). From 23 months after birth, spasms began to occur, which became severe when the sleep disorder happened frequently. At approximately age 4 , general myoclonus epilepsy occurred. Thereafter, she took valproic acid $125 \mathrm{mg}$, in divided into two doses two times per day, and experienced convulsion at 3 to 5 months intervals. An SMS (Social Maturity Scale) test, which was taken in the 30 month after birth, revealed severe intellectual retardation with a social age of 0.32 . Regarding motor development, she was equivalent to a 4-7 month old for the Gross motor, 1-4 month old for the Fine motor, and 1-2 months old for language development.

The neurological test revealed that the child's flexor in the arms and legs was hyper-tensed, her skeletal muscle was not fully developed, and she showed slight dystonic tremor. The morphological characteristics were microcephaly and hypoplasia of the maxillary bones. In the anteroom for day surgery, the child patient was uncooperative with the medical staff and would not leave her parent. The girl could not walk, and she was nestling in her parent's arms, speaking incomprehensible words and making infelicitous laughs. The blood test before the operation revealed a hemoglobin level of $11.8 \mathrm{~g} / \mathrm{dl}$, which was slightly low. However, there were no

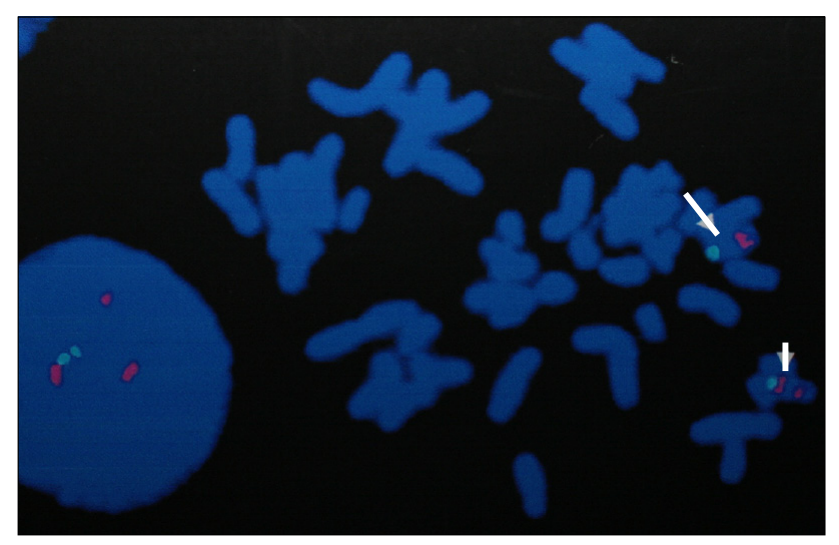

Fig. 1. FISH test of the patient. Arrowhead: normal chromosome 15; the red signal (SNRPN, small nuclear ribonucleoprotein polypeptide $\mathrm{N})$ below green signal (centromere [D15Z1] region), Arrow: del (15) (q11.2q13); no red signal (SNRPN, small nuclear ribonucleoprotein polypeptide N). abnormalities found in the electrocardiogram and chest X-ray.

In the anteroom for day surgery, an intravenous route was secured with the assistance of the surgical staff and parent. For the induction of general anesthesia, midazolam $2 \mathrm{mg}$ instead of the ketamine commonly used in the hospital was administered with concern over seizure. However, it failed to sedate her and another $2 \mathrm{mg}$ was administrated, which had no effects. A decision was made not to use different kinds of intravenous anesthetics because of the concern about the delay in awakening and restoration from anesthesia. The girl was carried into the operating room (OR) in her parent's arms. The vital signs immediately after entering the OR revealed a blood pressure, pulse rate and oxygen saturation of $110 / 60 \mathrm{mmHg}$, at $110 /$ min and $96 \%$, respectively. Sevoflurane and $100 \%$ oxygen were employed to induce anesthesia, and after administering rocuronium bromide $10 \mathrm{mg}$ as a muscle relaxant, mask ventilation began and there was no difficulty in ventilation. After confirming full muscle relaxation, an orotracheal intubation was performed with a cuffed endotracheal tube (4.5 mm) without problems. Expecting trouble in endotracheal intubation, oropharyngeal intubation was first performed and the operation proceeded while maintaining orotracheal intubation at the consent of the pediatric dentistry department. By regulating the oxygen to air ratio to set a Fraction of Inspired Oxygen $\left(\mathrm{FiO}_{2}\right)$ at 0.5 , controlled respiration was performed to maintain a partial carbon dioxide pressure in expiration of 30-35 mmHg while maintaining anesthesia with sevoflurane 3 vol\%. During surgery, the blood pressure and pulse oximetry was stable at 100-110/60-70 $\mathrm{mmHg}$ and $99-100 \%$, respectively. After surgery, spontaneous respiration was fully restored by manual ventilation. Pyridostigmine $5 \mathrm{mg}$ and glycopyrrolate $0.15 \mathrm{mg}$ were then administrated to reverse muscle relaxant. Before extubation, the Train of Four (TOF) stimuli was measured to indicate 3 and the endotracheal tube was removed after confirming that the muscle function had been fully restored. The total length of time for tooth conservation surgery was 100 minutes, and the length of anesthesia was 125 minutes. In the postanesthetic recovery unit, the patient showed stable breathing and vital signs and her motion functions were checked after reaching consciousness. She was discharged home without complications. The next day's follow-up phone call also confirmed her good general condition without significant complications.

\section{Discussion}

Angelman Syndrome (AS) is a congenital disorder showing a variety of clinic manifestations due to a partial deficit on the paired chromosome 15. The prevalence of AS is one in $10,000-40,000$ of the population and its clinical characteristics 
are so diverse that a diagnosis of AS rests on a combination of molecular genetic testing and/or cytogenetic analysis. $70 \%$ of AS patients have the loss of the maternally contributed region of Chromosome 15q11-13, while 15\% of AS have uniparental disomy coming from a paternal contribution. $60 \%$ of the paternal deletion in the chromosome region 15q11-13 may cause Prader-Willi Syndrome (PWS) [4]. The characteristic symptoms of PWS can be differentiated from those of AS by the clinical features, by hypotonicity and early obesity in infancy, mental retardation and hypogonadsm. Genetic imprinting refers to the accentuation or activation of genes that can be expressed in a parent-of-origin-specific manner. For the remaining $20 \%$ of AS cases, no genetic malformation is manifested but patients have a mutation of the UBE3A gene and show fewer abnormalities in the electroencephalographic (EEG) findings and a smaller number of spasms [5].

The clinic features of a genetic mutation and deficiency vary according to the gene expressions of RB3, which is critical in the genetic coding of the $\beta_{3}$ subunit of the GABA-Areceptor [6]. Many drugs acting on the central nervous system, such as antianxiety medication, sedative-hypnotics, general anesthetics, anti-seizure drugs, etc. are considered to acton the GABA receptor. A study on genetically manipulated mice of aminoacid mutated from the $\beta_{3}$ subunit of the GABA receptor showed that reduced effects of propofol and etomidate [7]. Homanics et al. [8] reported a meaningful decrease in benzodiazepine binding in genetically manipulated mouse models of Angelman syndrome. In the present case, midazolam $4 \mathrm{mg}$ was administered into the vein before surgery to reduce the patient's anxiety, but she was not sedated. Similarly, reciprocal interactions between the receptor region and intravenous anesthetics warrant further study. Sugimura et al. [9] and Gyulai et al. [10] revealed the influence of halogenated ether on the GABA receptors. A change in the distribution of the receptors caused by isoflurane and partly by sevoflurane activates the outer structure of the synapse through osmotic changes in ions. Such a polar change in neuronal cells not only restrains the activity of neuronal cells themselves but also reflect the state of the general anesthetics.

Therefore, it is reasonable to minimize or avoid administering an excessive dose of benzodiazepine to patients with this syndrome, and to refrain from using halogenated ethers as an inhalation anesthetic. From a theoretical viewpoint, intravenous anesthesia using a combination of propofol and fentanyl, which are not known to collide with the GABA system, will be safe [11].

On the other hand, patients with AS can show symptoms of separation anxiety due to their severe mental retardation. For child patients who show separation anxiety at general anesthesia for outpatient surgery, securing an intravenous route after admitting the patients into the operating room accompanied by their parents and sedating them with inhalation anesthetics is desirable for reducing the children's anxiety.

Patients with this syndrome usually require general anesthesia for orthopedic surgery for the skeletal system dysfunction or even in a simple operation because they are uncooperative [12]. AS may not be detected at birth or during infancy, and is revealed mainly after the age of four, when the characteristic behaviors and signs appear. With several exceptions, the life expectancy generally does not exceed 15 years $[3,4]$. Table 1

Table 1. Developmental and Physical Findings in Angelman Syndrome [13]

$$
\text { Consistent (100\%) }
$$

- Developmental delay, functionally severe

- Movement or balance disorder, usually ataxia of gait and/or tremulous movement of limbs. Movement disorder can be mild. May not appear as frank ataxia but can be forward lurching, unsteadiness, clumsiness, or quick, jerky motions

- Behavioral uniqueness: any combination of frequent laughter/ smiling; apparent happy demeanor; easily excitable personality, often with uplifted hand-flapping or waving movements; hypermotoric behavior

- Speech impairment, none or minimal use of words; receptive and non-verbal communication skills higher than verbal ones

Frequent (more than $80 \%$ )

- Delayed, disproportionate growth in head circumference, usually resulting in microcephaly ( $\leq 2$ S.D. of normal OFC) by age 2 years. Microcephaly is more pronounced in those with 15q11.2-q13 deletions.

- Seizures, onset usually $<3$ yrs. of age. Seizure severity usually decreases with age but the seizure disorder lasts throughout adulthood.

- Abnormal EEG, with a characteristic pattern, as mentioned in the text. The EEG abnormalities can occur in the first 2 years of life and can precede clinical features, and are often not correlated to clinical seizure events.

Associated (20-80\%)

- Flat occiput

- Occipital groove

- Protruding tongue

- Tongue thrusting; suck/swallowing disorders

- Feeding problems and/or truncal hypotonia during infancy

- Prognathia

- Wide mouth, wide-spaced teeth

- Frequent drooling

- Excessive chewing/mouthing behaviors

- Strabismus

- Hypopigmented skin, light hair and eye color (compared to family), seen only in deletion cases

- Hyperactive lower extremity deep tendon reflexes

- Uplifted, flexed arm position especially during ambulation

- Wide-based gait with pronated or valgus-positioned ankles

- Increased sensitivity to heat

- Abnormal sleep wake cycles and diminished need for sleep

- Attraction to/fascination with water; fascination with crinkly items such as certain papers and plastics

- Abnormal food related behaviors

- Obesity (in the older child)

- Scoliosis

- Constipation 
shows the consensus criteria of the clinical features in AS, enacted in 2005 [13].

Patients with AS do not have cardiovascular disorders or arrhythmia as common features but may have bradycardia due to hypertension of the vagus nerves. This phenomenon is noticed mainly during convulsive laughter, which can contribute to severe arrhythmia or ventricular asystole or fainting, resulted from an increase in the intrathoracic pressure and Valsalva effect. The administration of atropine to treat the unexpected arrhythmia during surgery is not always effective. Moreover, the immediate use of cardiac massage and epinephrine should be considered if asystole occurs [14].

Malformations of the skull and facial bones (microcephaly, mandibular bulging, etc.) found in patients with AS can be a serious hindrance to tracheal intubation. However, the present case did not experience significant difficulty, and the literature review has not reported any difficulty in tracheal intubation. However, preparations should be made for any possible contingency when an unexpected malformation is confronted.

Many patients with AS take medication for hyperactivity and epilepsy. To prevent perioperative epilepsy, it will be wise to keep taking anti-seizure drugs during the operation. However, it is unclear if all patients with AS should take antiseizure medication as preventive medicine. When choosing anesthetics, drugs that can cause seizure, such as ketamine or enflurane, should be avoided.

Peripheral muscular atrophy is another problem that needs to be considered when administrating anesthesia to patients with AS. When it does occur, the sensitivity may increase, and a smaller amount of muscle relaxant than the usual dosage should be applied. Monitoring the level of muscle relaxation is required to ensure the appropriateness in the degree of muscle relaxation during operation and during restoration from muscle relaxation after surgery. In the present case, the anesthesia was induced with inhalation anesthetics and maintained with a small dose of muscle relaxant, while there were no added muscle relaxants except those administered before the induction of anesthesia. TOF (Train of Four) stimuli indicated a count of 3 before extubation after surgery. The endotracheal tube was removed safely and spontaneous respiration resumed. In conclusion, monitoring of the muscle relaxation of patients is necessary because of the possible risks of seizures and peripheral muscular atrophy. In particular, for outpatient surgery, extra precautions against accidents, such as falls due to muscular atrophy, should be taken after being discharged home. In addition, preparations should be made for emergencies, such as arrhythmia and ventricular asystole due to the hypertension of the vagus nerves. The difficulty in securing the respiratory tract in cases with malformations of the facial bones should be anticipated. In addition, proper attention should be paid to the choice of intravenous anesthetics and their correct dose in an abnormal case of an impairment of the GABA receptors.

\section{References}

1. Angelman H. "Puppet" children. A report of three cases. Dev Med Child Neurol 1965; 7: 681-8.

2. Schneider BB, Maina DM. Angelman syndrome. J Am Optom Assoc 1993; 64: 502-6.

3. Hou JW, Wang PJ, Wang TR. Angelman syndrome assessed by neurological and molecular cytogenetic and molecular investigation. Pediatr Neurol 1997; 16: 17-22

4. Ebert MH, Schmidt DE, Thompson T, Butler MG. Elevated plasma gamma-aminobutyric acid (GABA) levels in individuals with either Prader-Willi syndrome or Angelman syndrome. J Neuropsychiatry Clin Neurosci 1997; 9: 75-80.

5. Moncla A, Malzac P, Voelckel MA, Auquier P, Girardot L, Mattei MG, et al. Phenotype-genotype correlation in 20 deletion and 20 non-deletion Angelman syndrome patients. Eur J Hum Genet 1999; 7: 131-9.

6. DeLorey TM, Handforth A, Anagnostaras SG, Homanics GE, Minassian BA, Asatourian A, et al. Mice lacking the beta3 subunit of the GABAA receptor have the epilepsy phenotype and many of the behavioral characteristics of Angelman syndrome. J Neurosci 1998; 18: 8505-14.

7. Jurd R, Arras M, Lambert S, Drexler B, Siegwart R, Crestani F, et al. General anesthetic actions in vivo strongly attenuated by a point mutation in the GABA (A) receptor beta3 subunit. FASEB J 2003; 17: 250-2.

8. Homanics GE, DeLorey TM, Firestone LL, Quinlan JJ, Handforth A, Harrison NL, et al. Mice devoid of gamma-aminobutyrate type A receptor beta3 subunit have epilepsy, cleft palate, and hypersensitive behavior. Proc Natl Acad Sci U S A 1997; 94: 4143-8.

9. Sugimura M, Kitayama S, Morita K, Irifune M, Takarada T, Kawahara M, et al. Effects of volatile and intravenous anesthetics on the uptake of GABA, glutamate and dopamine by their transporters heterologously expressed in COS cells and in rat brain synaptosomes. Toxicol Lett 2001; 123: 69-76.

10. Gyulai FE, Mintun MA, Firestone LL. Dose-dependent enhancement of in vivo GABA (A)-benzodiazepine receptor binding by isoflurane. Anesthesiology 2001; 95: 585-93.

11. Bujok G, Knapik P. Angelman syndrome as a rare anaesthetic problem. Paediatr Anaesth 2004; 14: 281-3.

12. Rösby O, Strömme P, Sandsmark M, Ramstad K, Ormerod E, Birger van der Hagen C, et al. Unilateral cleft lip in a boy with Angelman syndrome. J Craniofac Genet Dev Biol 1996; 16: 122-5.

13. Williams CA, Beaudet AL, Clayton-Smith J, Knoll JH, Kyllerman M, Laan LA, et al. Angelman syndrome 2005: updated consensus for diagnostic criteria. Am J Med Genet A 2006; 140: 413-8.

14. Vanagt WY, Pulles-Heintzberger CF, Vernooy K, Cornelussen RN, Delhaas T. Asystole during outbursts of laughing in a child with Angelman syndrome. Pediatr Cardiol 2005; 26: 866-8. 